Acta Crystallographica Section E

Structure Reports

Online

ISSN 1600-5368

\section{Howard A. Shallard-Brown,* David J. Watkin and Andrew R. Cowley}

Chemical Crystallography Laboratory, Chemistry Research Laboratory, Oxford University, Mansfield Road, Oxford OX1 3TA, England

Correspondence e-mail:

howard.shallard-brown@Imh.ox.ac.uk

\section{Key indicators}

Single-crystal X-ray study

$T=150 \mathrm{~K}$

Mean $\sigma(\mathrm{C}-\mathrm{C})=0.002 \AA$

$R$ factor $=0.040$

$w R$ factor $=0.104$

Data-to-parameter ratio $=21.7$

For details of how these key indicators were automatically derived from the article, see http://journals.iucr.org/e.

\title{
n-Butyl acetate
}

The title compound, $\mathrm{C}_{6} \mathrm{H}_{12} \mathrm{O}_{2}$, was prepared by a modified zone-refinement method at $150 \mathrm{~K}$ and consists of discrete molecules in van der Waals contact.

\section{Comment}

Many of the esters and ketones used in the flavours and fragrances industry are liquid at room temperature; thus, in the past, crystalline derivatives have had to be prepared for $\mathrm{X}$-ray analysis. As part of a programme to systematize in situ crystal growth from liquids, we have examined a range of commercially available chemicals. Low-molecular-weight organic esters are liquid at room temperature. To date, only the crystal structure of methyl acetate has been determined (Barrow et al., 1981). It was shown that the molecules of methyl acetate exist as discrete entities, without any strong intermolecular contacts. $n$-Butyl acetate, (I), was examined because it has a melting point suitable for our trials. The crystal structure is similar to that of methyl acetate, consisting of discrete molecules in van der Waals contact. The most evident feature is the pairwise parallel butyl residues related by a crystallographic centre of symmetry. The open packing of the structure is reflected in its low density of $1.09 \mathrm{Mg} \mathrm{m}^{-3}$.

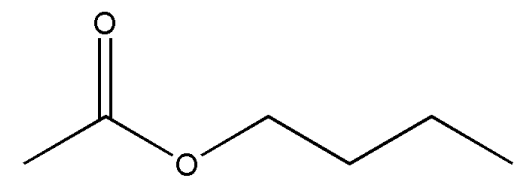

(I)

\section{Experimental}

A $3 \mathrm{~mm}$ column of the title material, which is a liquid at room temperature, was sealed in a $0.2 \mathrm{~mm}$ Lindemann tube, which was not accurately parallel to the $\varphi$ axis. A single crystal of the compound was grown by keeping the compound under a cold nitrogen gas stream at $150 \mathrm{~K}$ (a little below its melting point), and slowly moving a small

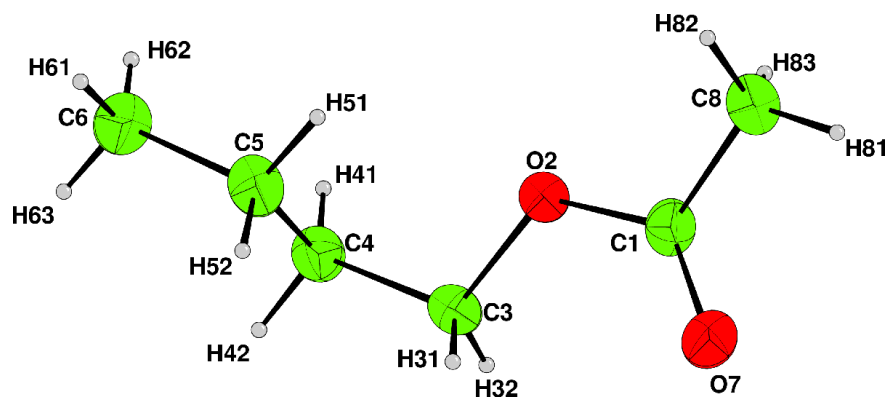

Figure 1

The title compound, with displacement ellipsoids drawn at the $50 \%$ probability level. $\mathrm{H}$ atoms are of arbitrary radii.
Received 16 December 2004 Accepted 5 January 2005 Online 5 February 2005
(C) 2005 International Union of Crystallography Printed in Great Britain - all rights reserved 
liquid zone, created by a micro-heating coil, up and down the sample. Once a suitable approximately single-crystal specimen had been obtained, the main data collection was carried out at this temperature. Because not all the data were collected with the Lindemann tube perpendicular to the X-ray beam, the multi-scan corrections applied by DENZO/SCALEPACK (Otwinowski \& Minor, 1997) also contain contributions due to changes in illuminated volume of the cylindrical sample; this is reflected in the ratio $T_{\min } / T_{\max }$.

$$
\begin{aligned}
& \text { Crystal data } \\
& \mathrm{C}_{6} \mathrm{H}_{12} \mathrm{O}_{2} \\
& M_{r}=116.16 \\
& \text { Triclinic, } P \overline{1} \\
& a=4.7272(1) \AA \\
& b=7.6955(3) \AA \\
& c=10.1387(4) \AA \\
& \alpha=100.7426(13)^{\circ} \\
& \beta=96.0038(15)^{\circ} \\
& \gamma=99.3371(18)^{\circ} \\
& V=354.09(2) \AA^{3}
\end{aligned}
$$

$$
Z=2
$$$$
D_{x}=1.089 \mathrm{Mg} \mathrm{m}^{-3}
$$

Mo $K \alpha$ radiation

Cell parameters from 1476

reflections

$\theta=5-27^{\circ}$

$\mu=0.08 \mathrm{~mm}^{-1}$

$T=150 \mathrm{~K}$

Cylinder, colourless

$0.80 \times 0.20 \times 0.20 \mathrm{~mm}$

\section{Data collection}

Nonius KappaCCD diffractometer $\omega$ scans

Absorption correction: multi-scan (DENZO/SCALEPACK; Otwinowski \& Minor, 1997)

$T_{\min }=0.33, T_{\max }=0.98$

5958 measured reflections

\section{Refinement}

Refinement on $F^{2}$

$R\left[F^{2}>2 \sigma\left(F^{2}\right)\right]=0.040$

$w R\left(F^{2}\right)=0.104$

$S=0.97$

1585 reflections

73 parameters
1585 independent reflections 1194 reflections with $I>2 \sigma(I)$

$R_{\text {int }}=0.035$

$\theta_{\max }=27.4^{\circ}$

$h=-6 \rightarrow 5$

$k=-9 \rightarrow 9$

$l=-13 \rightarrow 13$

$\mathrm{H}$-atom parameters constrained $w=1 /\left[\sigma^{2}(F)+0.045+0.061 P\right]$ where $P=\left[\max \left(F_{\mathrm{o}}{ }^{2}, 0\right)+2 F_{\mathrm{c}}{ }^{2}\right] / 3$ $(\Delta / \sigma)_{\max }<0.0001$

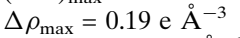

$\Delta \rho_{\min }=-0.31 \mathrm{e}^{-3}$
Table 1

Selected geometric parameters $\left(\AA{ }^{\circ}{ }^{\circ}\right)$.

\begin{tabular}{llll}
\hline $\mathrm{C} 1-\mathrm{O} 2$ & $1.3411(14)$ & $\mathrm{C} 3-\mathrm{C} 4$ & $1.5096(16)$ \\
$\mathrm{C} 1-\mathrm{O} 7$ & $1.2037(14)$ & $\mathrm{C} 4-\mathrm{C} 5$ & $1.5192(17)$ \\
$\mathrm{C} 1-\mathrm{C} 8$ & $1.4981(17)$ & $\mathrm{C} 5-\mathrm{C} 6$ & $1.5189(18)$ \\
$\mathrm{O} 2-\mathrm{C} 3$ & $1.4571(14)$ & & \\
$\mathrm{O} 2-\mathrm{C} 1-\mathrm{O} 7$ & $123.27(11)$ & $\mathrm{O} 2-\mathrm{C} 3-\mathrm{C} 4$ & $107.14(9)$ \\
$\mathrm{O} 2-\mathrm{C} 1-\mathrm{C} 8$ & $111.16(10)$ & $\mathrm{C} 3-\mathrm{C} 4-\mathrm{C} 5$ & $113.98(10)$ \\
$\mathrm{O} 7-\mathrm{C} 1-\mathrm{C} 8$ & $125.57(11)$ & $\mathrm{C} 4-\mathrm{C} 5-\mathrm{C} 6$ & $112.59(11)$ \\
$\mathrm{C} 1-\mathrm{O} 2-\mathrm{C} 3$ & $115.87(9)$ & & \\
\hline
\end{tabular}

All $\mathrm{H}$ atoms were located in a difference map, but those attached to $\mathrm{C}$ atoms were repositioned geometrically. The $\mathrm{H}$ atoms were initially refined with soft restraints on the bond lengths and angles to regularize their geometry $(\mathrm{C}-\mathrm{H}=0.97-1.01 \AA)$, after which they were refined with riding constraints and with $U_{\text {iso }}(\mathrm{H})=1.2 U_{\text {eq }}(\mathrm{C})$.

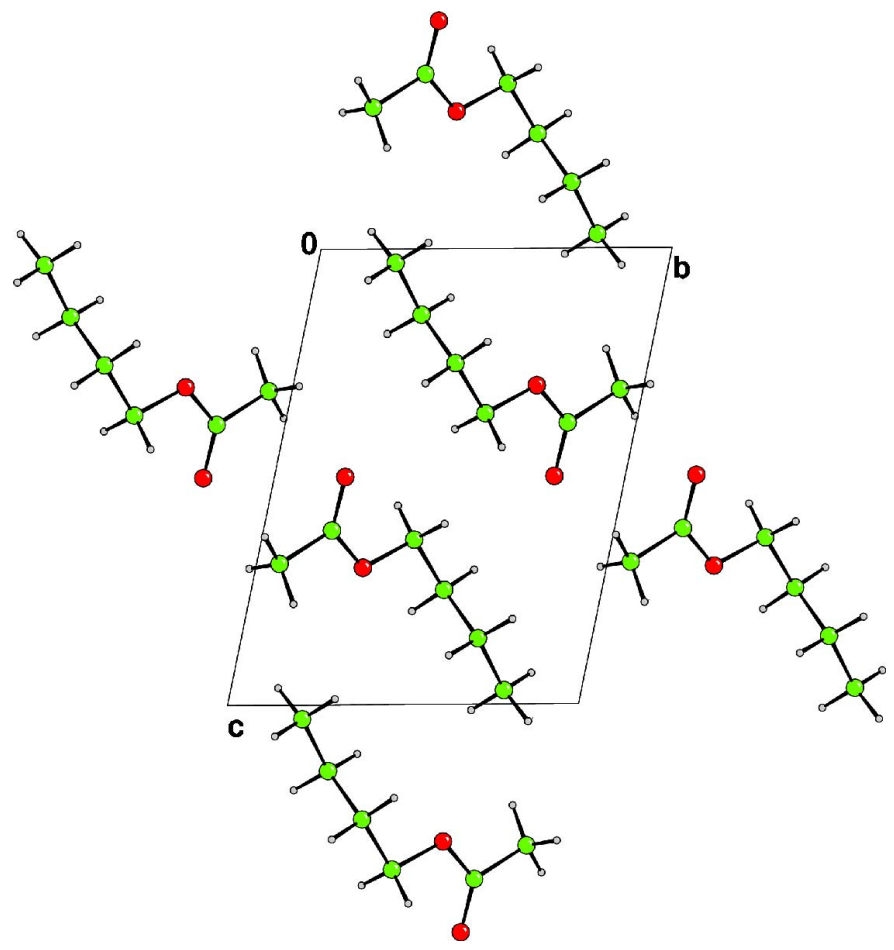

Figure 2

A packing diagram, viewed along the $a$ axis, showing the parallel pairing of the butyl groups.

Data collection: COLLECT (Nonius, 1997); cell refinement: DENZO/SCALEPACK (Otwinowski \& Minor, 1997); data reduction: $D E N Z O / S C A L E P A C K$; program(s) used to solve structure: SIR92 (Altomare et al., 1994); program(s) used to refine structure: CRYSTALS (Betteridge et al., 2003); molecular graphics: CAMERON (Watkin et al., 1996); software used to prepare material for publication: CRYSTALS.

\section{References}

Altomare, A., Cascarano, G., Giacovazzo, G., Guagliardi, A., Burla, M. C., Polidori, G. \& Camalli, M. (1994). J. Appl. Cryst. 27, 435.

Barrow, M. J., Cradock, S., Ebsworth, E. A. V. \& Rankin, D. W. H. (1981). J. Chem. Soc. Dalton Trans. 16, 1988-1993.

Betteridge, P. W., Carruthers, J. R., Cooper, R. I., Prout, K. \& Watkin, D. J. (2003). J. Appl. Cryst. 36, 1487.

Nonius (1997). COLLECT. Nonius BV, Delft, The Netherlands.

Otwinowski, Z. \& Minor, W. (1997). Methods in Enzymology, Vol. 276, Macromolecular Crystallography, Part A, edited by C. W. Carter Jr \& R. M. Sweet, pp. 307-326. New York: Academic Press.

Watkin, D. J., Prout, C. K. \& Pearce, L. J. (1996). CAMERON. Chemical Crystallography Laboratory, Oxford, England. 


\section{supporting information}

Acta Cryst. (2005). E61, o561-o562 [https://doi.org/10.1107/S1600536805000371]

\section{$n$-Butyl acetate}

Howard A. Shallard-Brown, David J. Watkin and Andrew R. Cowley

n-Butyl acetate

Crystal data

$\mathrm{C}_{6} \mathrm{H}_{12} \mathrm{O}_{2}$

$M_{r}=116.16$

Triclinic, $P \overline{1}$

$a=4.7272(1) \AA$

$b=7.6955(3) \AA$

$c=10.1387(4) \AA$

$\alpha=100.7426(13)^{\circ}$

$\beta=96.0038(15)^{\circ}$

$\gamma=99.3371(18)^{\circ}$

$V=354.09(2) \AA^{3}$

$Z=2$

$F(000)=128$

$D_{\mathrm{x}}=1.089 \mathrm{Mg} \mathrm{m}^{-3}$

Mo $K \alpha$ radiation, $\lambda=0.71073 \AA$

Cell parameters from 1476 reflections

$\theta=5-27^{\circ}$

$\mu=0.08 \mathrm{~mm}^{-1}$

$T=150 \mathrm{~K}$

Cylinder, colourless

$0.80 \times 0.20 \times 0.20 \mathrm{~mm}$

Data collection

Nonius KappaCCD

5958 measured reflections diffractometer

Graphite monochromator

$\omega$ scans

1585 independent reflections

1194 reflections with $I>2 \sigma(I)$

Absorption correction: multi-scan

(DENZO/SCALEPACK; Otwinowski \& Minor,

$R_{\text {int }}=0.035$

$\theta_{\max }=27.4^{\circ}, \theta_{\min }=5.1^{\circ}$ 1997)

$T_{\min }=0.33, T_{\max }=0.98$

$h=-6 \rightarrow 5$

$k=-9 \rightarrow 9$

$l=-13 \rightarrow 13$

Refinement

Refinement on $F^{2}$

Least-squares matrix: full

$R\left[F^{2}>2 \sigma\left(F^{2}\right)\right]=0.040$

$w R\left(F^{2}\right)=0.104$

$S=0.97$

1585 reflections

73 parameters

0 restraints

Primary atom site location: structure-invariant direct methods

Hydrogen site location: inferred from neighbouring sites

$\mathrm{H}$-atom parameters constrained

$w=1 /\left[\sigma^{2}(F)+0.045+0.061 P\right]$

where $P=\left[\max \left(F_{\mathrm{o}}^{2}, 0\right)+2 F_{\mathrm{c}}^{2}\right] / 3$

$(\Delta / \sigma)_{\max }=0.0002$

$\Delta \rho_{\max }=0.19 \mathrm{e} \AA^{-3}$

$\Delta \rho_{\min }=-0.31 \mathrm{e}^{-3}$

Fractional atomic coordinates and isotropic or equivalent isotropic displacement parameters $\left(\hat{A}^{2}\right)$

\begin{tabular}{lllll}
\hline & $x$ & $y$ & $z$ & $U_{\text {iso }} * / U_{\text {eq }}$ \\
\hline C1 & $0.4329(2)$ & $0.19532(15)$ & $0.61673(12)$ & 0.0319 \\
O2 & $0.65680(17)$ & $0.30569(11)$ & $0.69916(8)$ & 0.0339 \\
C3 & $0.8300(3)$ & $0.43555(16)$ & $0.63810(12)$ & 0.0346
\end{tabular}




$\begin{array}{lllll}\text { C4 } & 1.0599(3) & 0.54983(16) & 0.74868(12) & 0.0343 \\ \text { C5 } & 0.9433(3) & 0.67482(17) & 0.85537(13) & 0.0391 \\ \text { C6 } & 1.1774(3) & 0.77932(18) & 0.96950(14) & 0.0473 \\ \text { O7 } & 0.37156(19) & 0.20225(12) & 0.49981(9) & 0.0425 \\ \text { C8 } & 0.2750(3) & 0.06562(18) & 0.68942(14) & 0.0422 \\ \text { H31 } & 0.7054 & 0.5128 & 0.6039 & 0.0396^{*} \\ \text { H32 } & 0.9191 & 0.3691 & 0.5635 & 0.0386^{*} \\ \text { H41 } & 1.1621 & 0.4712 & 0.7951 & 0.0409^{*} \\ \text { H42 } & 1.2056 & 0.6241 & 0.7048 & 0.0407^{*} \\ \text { H51 } & 0.7923 & 0.6020 & 0.8923 & 0.0463^{*} \\ \text { H52 } & 0.8546 & 0.7619 & 0.8135 & 0.0456^{*} \\ \text { H61 } & 1.0958 & 0.8659 & 1.0380 & 0.0562^{*} \\ \text { H62 } & 1.2725 & 0.6978 & 1.0129 & 0.0569^{*} \\ \text { H63 } & 1.3283 & 0.8469 & 0.9317 & 0.0556^{*} \\ \text { H81 } & 0.0981 & 0.0075 & 0.6299 & 0.0492^{*} \\ \text { H82 } & 0.2414 & 0.1286 & 0.7779 & 0.0492^{*} \\ \text { H83 } & 0.3985 & -0.0178 & 0.6998 & 0.0499^{*}\end{array}$

Atomic displacement parameters $\left(\AA^{2}\right)$

\begin{tabular}{lllllll}
\hline & $U^{11}$ & $U^{22}$ & $U^{33}$ & $U^{12}$ & $U^{13}$ & $U^{23}$ \\
\hline C1 & $0.0323(6)$ & $0.0278(6)$ & $0.0334(7)$ & $0.0043(5)$ & $0.0010(5)$ & $0.0040(5)$ \\
O2 & $0.0365(5)$ & $0.0326(5)$ & $0.0297(5)$ & $-0.0025(4)$ & $0.0006(3)$ & $0.0092(4)$ \\
C3 & $0.0359(6)$ & $0.0347(6)$ & $0.0330(6)$ & $-0.0005(5)$ & $0.0047(5)$ & $0.0123(5)$ \\
C4 & $0.0310(6)$ & $0.0349(7)$ & $0.0361(7)$ & $0.0018(5)$ & $0.0024(5)$ & $0.0096(5)$ \\
C5 & $0.0390(7)$ & $0.0339(7)$ & $0.0432(7)$ & $0.0028(5)$ & $0.0060(6)$ & $0.0078(6)$ \\
C6 & $0.0593(9)$ & $0.0387(8)$ & $0.0385(7)$ & $0.0000(6)$ & $0.0018(6)$ & $0.0051(6)$ \\
O7 & $0.0461(5)$ & $0.0411(5)$ & $0.0350(5)$ & $-0.0011(4)$ & $-0.0061(4)$ & $0.0092(4)$ \\
C8 & $0.0460(7)$ & $0.0353(7)$ & $0.0410(7)$ & $-0.0034(6)$ & $0.0044(6)$ & $0.0072(6)$ \\
\hline
\end{tabular}

Geometric parameters $\left(\AA,{ }^{\circ}\right)$

\begin{tabular}{llll}
\hline $\mathrm{C} 1-\mathrm{O} 2$ & $1.3411(14)$ & $\mathrm{C} 5-\mathrm{C} 6$ & $1.5189(18)$ \\
$\mathrm{C} 1-\mathrm{O} 7$ & $1.2037(14)$ & $\mathrm{C} 5-\mathrm{H} 51$ & 0.983 \\
$\mathrm{C} 1-\mathrm{C} 8$ & $1.4981(17)$ & $\mathrm{C} 5-\mathrm{H} 52$ & 0.983 \\
$\mathrm{O} 2-\mathrm{C} 3$ & $1.4571(14)$ & $\mathrm{C} 6-\mathrm{H} 61$ & 1.021 \\
$\mathrm{C} 3-\mathrm{C} 4$ & $1.5096(16)$ & $\mathrm{C} 6-\mathrm{H} 62$ & 0.971 \\
$\mathrm{C} 3-\mathrm{H} 31$ & 0.984 & $\mathrm{C} 6-\mathrm{H} 63$ & 0.968 \\
$\mathrm{C} 3-\mathrm{H} 32$ & 1.002 & $\mathrm{C} 8-\mathrm{H} 81$ & 0.967 \\
$\mathrm{C} 4-\mathrm{C} 5$ & $1.5192(17)$ & $\mathrm{C} 8-\mathrm{H} 82$ & 0.978 \\
$\mathrm{C} 4-\mathrm{H} 41$ & 0.988 & $\mathrm{C} 8-\mathrm{H} 83$ & 0.948 \\
$\mathrm{C} 4-\mathrm{H} 42$ & 1.015 & & \\
& & & 108.3 \\
$\mathrm{O} 2-\mathrm{C} 1-\mathrm{O} 7$ & $123.27(11)$ & $\mathrm{C} 4-\mathrm{C} 5-\mathrm{H} 51$ & 109.8 \\
$\mathrm{O} 2-\mathrm{C} 1-\mathrm{C} 8$ & $111.16(10)$ & $\mathrm{C} 6-\mathrm{C} 5-\mathrm{H} 51$ & 109.8 \\
$\mathrm{O} 7-\mathrm{C} 1-\mathrm{C} 8$ & $125.57(11)$ & $\mathrm{C} 4-\mathrm{C} 5-\mathrm{H} 52$ & 108.2 \\
$\mathrm{C} 1-\mathrm{O} 2-\mathrm{C} 3$ & $115.87(9)$ & $\mathrm{C} 6-\mathrm{C} 5-\mathrm{H} 52$ & \\
$\mathrm{O} 2-\mathrm{C} 3-\mathrm{C} 4$ & $107.14(9)$ & $\mathrm{H} 51-\mathrm{C} 5-\mathrm{H} 52$ &
\end{tabular}




$\begin{array}{llll}\mathrm{O} 2-\mathrm{C} 3-\mathrm{H} 31 & 109.0 & \mathrm{C} 5-\mathrm{C} 6-\mathrm{H} 61 & 111.2 \\ \mathrm{C} 4-\mathrm{C} 3-\mathrm{H} 31 & 109.1 & \mathrm{C} 5-\mathrm{C} 6-\mathrm{H} 62 & 110.7 \\ \mathrm{O} 2-\mathrm{C} 3-\mathrm{H} 32 & 109.0 & \mathrm{H} 61-\mathrm{C} 6-\mathrm{H} 62 & 111.4 \\ \mathrm{C} 4-\mathrm{C} 3-\mathrm{H} 32 & 111.0 & \mathrm{C} 5-\mathrm{C} 6-\mathrm{H} 63 & 109.1 \\ \mathrm{H} 31-\mathrm{C} 3-\mathrm{H} 32 & 111.3 & \mathrm{H} 61-\mathrm{C} 6-\mathrm{H} 63 & 109.3 \\ \mathrm{C} 3-\mathrm{C} 4-\mathrm{C} 5 & 113.98(10) & \mathrm{H} 62-\mathrm{C} 6-\mathrm{H} 63 & 104.9 \\ \mathrm{C} 3-\mathrm{C} 4-\mathrm{H} 41 & 109.5 & \mathrm{C} 1-\mathrm{C} 8-\mathrm{H} 81 & 106.2 \\ \mathrm{C} 5-\mathrm{C} 4-\mathrm{H} 41 & 107.8 & \mathrm{C} 1-\mathrm{C} 8-\mathrm{H} 82 & 110.4 \\ \mathrm{C} 3-\mathrm{C} 4-\mathrm{H} 42 & 108.1 & \mathrm{H} 81-\mathrm{C} 8-\mathrm{H} 82 & 113.2 \\ \mathrm{C} 5-\mathrm{C} 4-\mathrm{H} 42 & 109.2 & \mathrm{C} 1-\mathrm{C} 8-\mathrm{H} 83 & 105.1 \\ \mathrm{H} 41-\mathrm{C} 4-\mathrm{H} 42 & 108.1 & \mathrm{H} 81-\mathrm{C} 8-\mathrm{H} 83 & 110.9 \\ \mathrm{C} 4-\mathrm{C} 5-\mathrm{C} 6 & 112.59(11) & \mathrm{H} 82-\mathrm{C} 8-\mathrm{H} 83 & 110.7\end{array}$

